\title{
Hemodynamic Changes Following Endotracheal Intubation in Patients Undergoing Cesarean Section With General Anesthesia: Application of Glidescope $®$ Videolaryngoscope Versus Direct Laryngoscope
}

\author{
Shahram Amini ${ }^{1, *}$; Majid Shakib ${ }^{2}$ \\ ${ }^{1}$ Department of Anesthesiology and Critical Care, Mashhad University of Medical Sciences, Mashhad, Iran \\ $2_{\text {Buali Hospital, Artesh University of Medical Sciences, Birjand, Iran }}$ \\ *Corresponding author: Shahram Amini, Department of Anesthesiology and Critical Care, Mashhad University of Medical Sciences, Mashhad, Iran. Tel: +98-5138640535, E-mail: amin- \\ ish@mums.ac.ir
}

Received: July 7, 2014; Revised: August 16, 2014; Accepted: October 25, 2014

\begin{abstract}
Background: Endotracheal intubation is usually associated with hemodynamic changes, especially in patients undergoing cesarean section by general anesthesia. GlideScope® videolaryngoscope (GVL) is a novel video laryngoscope, which does not need direct exposure of vocal cords and produces lesser hemodynamic changes due to lower degrees of trauma and stimuli to oropharynx than the Macintosh direct laryngoscope(MDL).

Objectives:The aim of this study was to compare hemodynamic changes following endotracheal intubation with GVLand MDL in patients undergoing cesarean section by general anesthesia.

Materials and Methods: Seventy patients undergoing elective cesarean section by general anesthesia requiring endotracheal intubation were randomly allocated to be intubated with either GVL $(n=35)$ or MDL $(n=35)$. Systolic, diastolic and mean arterial blood pressure (MAP), as well as pulse rates, and rate pressure product (RPP) were compared at baseline, after induction of anesthesia, and after intubation at oneminute interval for five minutes between the two groups. The patients were also compared for Mallampati score, sore throat, intubation time and neonates' Apgar scores.

Results: The patients were similar regarding systolic, diastolic and mean arterial blood pressure. Pulse rate changes were significantly lower only at 1 and 3 minutes in the GVL group. The intubation times were $9.3 \pm 1.4$ and $10.6 \pm 1.7$ seconds in the MDL and GVL groups, respectively $(\mathrm{P}>0.05)$. RPP was also lower in the GVL group at 1 and 2 minutes $(\mathrm{P}<0.05)$ and returned to baseline afterwards. There was no significant difference between the groups for Mallampati score, sore throat and Apgar scores.

Conclusions: Our study revealed that hemodynamic parameters with GVL are only better preserved in the first three minutes after intubation in patients undergoing elective cesarean section and patients are similar regarding intubation time, sore throat and Apgar score.
\end{abstract}

Keywords: Glidescope; Endotracheal Intubation; Cesarean Section; Hemodynamics; Laryngoscope

\section{Background}

Circulatory response following endotracheal intubation can be significant and results in adverse outcomes $(1,2)$. Several drugs are currently used to blunt these effects (3, 4); however, they might have deleterious effects on hemodynamics. On the other hand, drugs such as opioids are not usually used for blunting circulatory response following endotracheal intubation in parturient due to their adverse effects on neonates. Glidescope $₫$ videolaryngoscope (GVL, Glidescope $®$, Saturn Biomedical Systems Inc., Canada) is a relatively novel device used for both oral and nasal endotracheal intubation in patients with normal and difficult airways (5-7). It has been associated with high success rates and minor complications even in hands of novices (8). Unlike a Macintosh direct laryngoscope (MDL), GVL with a specially designed blade and a $60^{\circ}$ curvature could decrease the upward lifting force and cervi- cal spine motion required to expose the glottis that might be associated with less hemodynamic changes. However, it is reported that GVL had no significant advantage over MDL in attenuating circulatory responses to orotracheal intubation in normotensive patients (9-12).

\section{Objectives}

The purpose of this study was to compare GVL and MDL for endotracheal intubation in patients undergoing cesarean section with general anesthesia. The primary outcome was to compare systolic, diastolic and mean arterial blood pressure (MAP). The secondary outcome was comparison of pulse rates, rate pressure product, Mallampati score, sore throat, intubation time and the Apgar scores between the two groups.

Copyright ( 2015, Iranian Society of Regional Anesthesia and Pain Medicine(ISRAPM). This is an open-access article distributed under the terms of the Creative Commons Attribution-NonCommercial 4.0 International License (http://creativecommons.org/licenses/by-nc/4.0/) which permits copy and redistribute the material just in noncommercial usages, provided the original work is properly cited. 


\section{Materials and Methods}

After approval from the ethics committee of our institution and obtaining an informed consent from participants, seventy parturients with ASA class of 1 or 2 admitted for elective cesarean section by general anesthesia were recruited for the study. The exclusion criteria were hypertension, predicted difficult airway, history of drug abuse, dehydration, history of any other cardiovascular diseases, history of consumption of any drugs known to affect the cardiovascular system and diabetes mellitus. Under a standard anesthesia, patients were randomly intubated with either GVL or MDL using a computer based system by a single experienced resident of anesthesiology. After preoxygenation with $100 \%$ oxygen for three minutes, anesthesia was induced with propofol $1.5 \mathrm{mg}$ $\mathrm{kg}$ and succinylcholine $1.5 \mathrm{mg} / \mathrm{kg}$ was given to facilitate endotracheal intubation. Anesthesia was maintained with 50\% nitrous oxide in oxygen and propofol $4 \mathrm{mg} . \mathrm{kg}$ ${ }^{1} \cdot \mathrm{h}^{-1}$ before the delivery. The patients received midazolam $0.02 \mathrm{mg} / \mathrm{kg}$ and fentanyl $2 \mu \mathrm{g} / \mathrm{kg}$ after the delivery. Anesthesia was maintained with $70 \%$ nitrous oxide in oxygen and propofol 4-6 mg. $\mathrm{kg}^{-1} \cdot \mathrm{h}^{-1}$ to maintain pulse rates and mean arterial blood pressure at $20 \%$ of the baseline values. The intubation time, defined as the time from grasping the endotracheal tube until observing a square wave on the capnograph, was recorded. Noninvasive mean arterial pressure (MAP), pulse rates (using electrocardiography monitoring) and rate pressure product (RPP) were recorded at baseline, after induction of anesthesia and every minute for five minutes after intubation. Patients were evaluated for development of sore throat before discharge from recovery room (graded as none, mild, moderate and severe) and neonate's Apgar score.

We considered a difference of $20 \%$ in MAP as clinically significant. A sample size of 28 was required to detect such a difference between the groups for a power of $80 \%$ at a significance level of $5 \%$. We recruited 35 patients in each group for possible missing. Arithmetic mean and standard variation values for different variables were calculated and statistical analyses were performed using SPSS software for Windows, version 15 (SPSS Inc., Chicago, IL, USA). We used independent student t-tests to compare continuous variables with normal distribution, repeated measure ANOVA for changes within the groups, and chisquared or Fisher's exact test for non-continuous variables. $\mathrm{P}<0.05$ was considered significant.

\section{Results}

The patients were similar regarding demographic data including age, weight, height and body mass index (BMI) (Table 1). Mallampati scores were similar in the both groups (Table 1). The intubation time was shorter in MDL group than GVL group; however, the difference was not significant ( $9.30 \pm 1.40$ seconds $v s .10 .60 \pm 1.70 ; \mathrm{P}=0.68$ ). The patients were similar regarding systolic, diastolic and mean arterial pressure at baseline. Systolic blood pres- sure was significantly lower at 1 minute after intubation in the GVL group $(\mathrm{P}=0.02)$. However, it was not different significantly. The patients were not different regarding diastolic and mean arterial pressure after intubation (Table 2). Pulse rates were significantly higher at 1, 2 and 3 minutes after intubation in MDL. Nevertheless, it returned to baseline after four minutes (Table 3). RPP was similar at baseline and after induction in the both groups. It was significantly lower in the GVL group at 1 and 2 minutes after intubation and returned to baseline with no significant difference after 3 minutes. There was no significant difference between the groups regarding sore throat (Table 4 ). The Apgar scores were $8.12 \pm 1.5$ and $8.74 \pm 0.67$ in the MDL and GVL groups, respectively $(\mathrm{P}=0.12)$.

\begin{tabular}{lccc}
\hline \multicolumn{4}{l}{ Table 1. Patients' Characteristics a,b } \\
\hline & The MDL & The GVL & P Value \\
\hline Age, $\mathbf{y}$ & $30.8 \pm 8.3$ & $29.7 \pm 7.3$ & 0.656 \\
Weight, kg & $71.0 \pm 8.2$ & $68.7 \pm 7.9$ & 0.286 \\
Height, cm & $162.6 \pm 4.8$ & $164.8 \pm 8.2$ & 0.609 \\
Mallampati grade 1 & $13(37.1)$ & $17(48.6)$ & 0.408 \\
Mallampati grade 2 & $22(62.9)$ & $18(51.4)$ & \\
\hline
\end{tabular}

a Abbreviations:GVL, GlideScope® videolaryngoscope; MDL, Macintosh direct laryngoscope.

$\mathrm{b}$ Values are presented as Mean \pm SD and No. (\%).

Table 2. Mean Arterial Pressures at Baseline, After Induction and After Intubation in the Study Groups a,b

\begin{tabular}{lccc}
\hline Timing & The MDL & The GVL & PValue \\
\hline Basic value, mm Hg & $92.5 \pm 7.0$ & $94.7 \pm 19.7$ & 0.431 \\
\hline After induction, mm Hg & $84.9 \pm 14.8$ & $86.2 \pm 16.2$ & 0.503 \\
\hline 1 Minute after intubation & $89.1 \pm 11.8$ & $91.8 \pm 13.4$ & 0.309 \\
\hline 2 Minutes after intubation & $85.9 \pm 7.3$ & $85.0 \pm 9.6$ & 0.743 \\
\hline 3 Minutes after intubation & $85.8 \pm 8.3$ & $85.1 \pm 9.1$ & 0.791 \\
\hline 4 Minutes after intubation & $84.1 \pm 6.8$ & $84.7 \pm 11.7$ & 0.778 \\
\hline 5 Minutes after intubation & $83.5 \pm 13.8$ & $84.6 \pm 10.6$ & 0.426 \\
\hline
\end{tabular}

a For abbreviations please refer to table 1 footnote.

$\mathrm{b}$ Values are presented as mean \pm SD.

Table 3. Pulse Rates at Baseline, After Induction and After Intubation in the Study Groups a,b

\begin{tabular}{lccc}
\hline Timing & The MDL & The GVL & PValue \\
\hline Basic value & $102.7 \pm 13.7$ & $98.5 \pm 11.9$ & 0.316 \\
\hline After induction & $95.9 \pm 20.4$ & $93.1 \pm 13.5$ & 0.482 \\
\hline 1 Minute after intubation & $98.6 \pm 19.3$ & $95.7 \pm 9.6$ & 0.298 \\
\hline Minutes after intubation & $92.7 \pm 11.5$ & $88.1 \pm 8.2$ & 0.211 \\
\hline Minutes after intubation & $88.2 \pm 9.1$ & $83.8 \pm 10.1$ & 0.155 \\
\hline 4 Minutes after intubation & $85.1 \pm 7.4$ & $82.0 \pm 11.1$ & 0.279 \\
\hline 5 Minutes after intubation & $83.5 \pm 9.3$ & $83.6 \pm 10.3$ & 0.743 \\
\hline $\begin{array}{l}\text { a For abbreviations please refer to table } 1 \text { footnote. } \\
\text { b values are presented as mean } \pm \text { SD. }\end{array}$ & &
\end{tabular}


Amini S et al.

Table 4. Incidence of Sore Throat in the Two Groups a,b

\begin{tabular}{lccc}
\hline Grade & The MDL & The GVL & P Value \\
\hline None & 20 & 22 & 0.75 \\
Mild & 12 & 10 & 0.48 \\
Moderate & 3 & 3 & 0.87 \\
\hline
\end{tabular}

${ }^{\mathrm{a}}$ For abbreviations please refer to table 1 footnote.

$\mathrm{b}$ Data are presented as numbers.

\section{Discussion}

Our study revealed that hemodynamic changes following endotracheal intubation are similar after three minutes with GVL and MDL in patients undergoing elective cesarean section with no significant difference on Apgar scores and sore throat. Significant hypertension and tachycardia are associated with tracheal intubation under light anesthesia. The magnitude of response is greater with increasing the force and duration of laryngoscopy. Although these changes are usually transient returning to control levels within five minutes, they can result in myocardial ischemia in patients with cardiac disease. Hemodynamic responses to tracheal intubation are mainly due to the stimulation of oropharyngeal structures produced by laryngoscopy and stimuli to larynx and trachea exerted by tracheal tube insertion. The upward lifting force required to expose the glottis during laryngoscopy is much less with glidescope compared to the Macintosh laryngoscope (4.9-13.7 N vs. 35-47.6 N) and results in less traction applied to soft tissues. Therefore, it might be associated with less sympathetic stimulation.

Blunting adverse effects of endotracheal intubation and provision of a rapid technique are still a dilemma in parturients. Increasing the depth of anesthesia can blunt these deleterious effects; however, changes in concentration of anesthetic agents in blood and at effector sites occur slowly in relation to the onset and offset of airway stimuli and hemodynamic responses. In addition, pharmacologic intervention such as opioids might depress cardiovascular system resulting in undesired hypotension and bradycardia. Besides, it can have deleterious respiratory effects on fetus. Therefore, it is generally suggested to administer opioids after the delivery. Another approach to reduce cardiovascular response to tracheal intubation is to modify the technique of tracheal intubation.

It has been suggested that general anesthesia of clinical standard depth can effectively suppress the pressor response, but not the tachycardia response to orotracheal intubation using a Glidescope (7). Although our patients demonstrated better hemodynamic effects in the first few minutes after intubation in the GVL group, parameters returned to baseline after 4 minutes in the both groups, which is not of clinical importance. Furthermore, these changes did not affect fetus. Our results are similar to those of Dashti et al. who demonstrated better hemodynamic responses with GVL than MDL in untreated pa- tients with hypertension (13). This in contrast to Xue et al. and Zhang et al. $(9,10)$ and Pournajafian et al. (14) who demonstrated similar hemodynamic stability following oral endotracheal intubation using GVL than MD group. Furthermore, Zhang et al. demonstrated similar hemodynamic response to orotracheal intubation with either fiberoptic broncoscope or GlideScope videolaryngoscope (10). Similarly, the pressor response to nasal tracheal intubation (NTI) has been reported to be similar with GSVL and MDL. Nevertheless, the tachycardia response to NTI was less and of a shorter duration when using GSVL than MDL (15).

In summary, we concluded that endotracheal intubation is associated with similar hemodynamic changes and fetal outcome with GVL and MDL in parturients undergoing cesarean section.

\section{Acknowledgements}

We would like to appreciate the operating room staff of Imam Ali Hospital, Zahedan, Iran, for their contribution in this study.

\section{Authors' Contributions}

Shahram Amini was responsible for study concept and design, analysis and interpretation of data, drafting of the manuscript; critical revision of the manuscript for important intellectual content, statistical analysis, administrative, technical and material support, study supervision. Majid Shakib was responsible for study concept and design, acquisition of data.

\section{References}

1. Beyer K, Taffe P, Halfon P, Pittet V, Pichard S, Haller G, et al. Hypertension and intra-operative incidents: a multicentre study of 125,000 surgical procedures in Swiss hospitals. Anaesthesia. 2009;64(5):494-502.

2. Goldman L, Caldera DL. Risks of general anesthesia and elective operation in the hypertensive patient. Anesthesiology. 1979;50(4):285-92.

3. Helfman SM, Gold MI, DeLisser EA, Herrington CA. Which drug prevents tachycardia and hypertension associated with tracheal intubation: lidocaine, fentanyl, or esmolol? Anesth Analg. 1991;72(4):482-6.

4. Omote K, Kirita A, Namiki A, Iwasaki H. Effects of nicardipine on the circulatory responses to tracheal intubation in normotensive and hypertensive patients. Anaesthesia. 1992;47(1):24-7.

5. Huang WT, Huang CY, Chung YT. Clinical comparisons between GlideScope video laryngoscope and Trachlight in simulated cervical spine instability. J Clin Anesth. 2007;19(2):110-4.

6. Malik MA, Maharaj CH, Harte BH, Laffey JG. Comparison of Macintosh, Truview EVO2, Glidescope, and Airwayscope laryngoscope use in patients with cervical spine immobilization. Br J Anaesth. 2008;101(5):723-30.

7. Xue FS, Zhang GH, Liu J, Li XY, Yang QY, Xu YC, et al. The clinical assessment of Glidescope in orotracheal intubation under general anesthesia. Minerva Anestesiol. 2007;73(9):451-7.

8. Kaki AM, Almarakbi WA, Fawzi HM, Boker AM. Use of Airtraq, CMac, and Glidescope laryngoscope is better than Macintosh in novice medical students' hands: A manikin study. Saudi J Anaesth. 2011;5(4):376-81.

9. Xue FS, Xu YC, Liu QJ, Yang QY, Liu Y, Liao X, et al. Hemodynamic 
responses to tracheal intubation with the Glidescope videolaryngoscope: a comparison of oral and nasal routes. Acta Anaesthesiol Taiwan. 2008;46(1):8-15.

10. Zhang GH, Xue FS, Li CW, Sun HY, Li XY, Sun HT, et al. [Comparison of hemodynamic responses to orotracheal intubation between using GlideScope videolaryngoscope and fiberoptic bronchoscope]. Zhongguo Yi Xue Ke Xue Yuan Xue Bao. 2006;28(3):406-9.

11. Xue FS, Zhang GH, Li XY, Sun HT, Li P, Sun HY, et al. Comparison of haemodynamic responses to orotracheal intubation with GlideScope videolaryngoscope and fibreoptic bronchoscope. Eur J Anaesthesiol. 2006;23(6):522-6.

12. Xue FS, Zhang GH, Li XY, Sun HT, Li P, Li CW, et al. Comparison of hemodynamic responses to orotracheal intubation with the GlideScope videolaryngoscope and the Macintosh direct laryn- goscope. J Clin Anesth. 2007;19(4):245-50.

13. Dashti M, Amini S, Azarfarin R, Totonchi Z, Hatami M. Hemodynamic changes following endotracheal intubation with Glidescope $®$ Video-laryngoscope in patients with untreated hypertension. Res Cardiovas Med. 2014;3(2);e21836.

14. Pournajafian AR, Ghodraty MR, Faiz SH, Rahimzadeh P, Goodarzynejad H, Dogmehchi E. Comparing GlideScope Video Laryngoscope and Macintosh Laryngoscope Regarding Hemodynamic Responses During Orotracheal Intubation: A Randomized Controlled Trial. Iran Red Crescent Med J. 2014;16(4):e21836.

15. Xue FS, Li XY, Liu QJ, Liu HP, Yang QY, Xu YC, et al. Circulatory responses to nasotracheal intubation: comparison of GlideScope videolaryngoscope and Macintosh direct laryngoscope. Chin Med J (Engl). 2008;121(14):1290-6. 